\title{
Biomechanical Stress and Strain Analysis of Mandibular Human Region from Computed Tomography to Custom Implant Development
}

\author{
Rafael Ferreira Gregolin, ${ }^{1}$ Cecília Amelia de Carvalho Zavaglia, ${ }^{2}$ \\ Ruís Camargo Tokimatsu, ${ }^{3}$ and João A. Pereira ${ }^{3}$ \\ ${ }^{1}$ Mechanical Engineering Department, Federal University of Grande Dourados (UFGD), Rodovia Dourados-Itahum, \\ Km 12, Cidade Universitária, Cx. Postal 533, 79804-970 Dourados, MS, Brazil \\ ${ }^{2}$ Mechanical Engineering Department, State University of Campinas (UNICAMP), Cidade Universitária Zeferino Vaz, \\ Barão Geraldo, 13083-970 Campinas, SP, Brazil \\ ${ }^{3}$ Mechanical Engineering Department, State University Paulista (UNESP), Avenida Brasil, No. 56, Centro, \\ 15385-000 Ilha Solteira, SP, Brazil \\ Correspondence should be addressed to Rafael Ferreira Gregolin; rfgregolin@gmail.com
}

Received 13 January 2017; Revised 14 March 2017; Accepted 20 March 2017; Published 23 April 2017

Academic Editor: Charles C. Sorrell

Copyright (C) 2017 Rafael Ferreira Gregolin et al. This is an open access article distributed under the Creative Commons Attribution License, which permits unrestricted use, distribution, and reproduction in any medium, provided the original work is properly cited.

Currently computational tools are helping and improving the processes and testing procedures in several areas of knowledge. Computed tomography (CT) is a diagnostic tool already consolidated and now beginning to be used as a tool for something even more innovative, creating biomodels. Biomodels are anatomical physical copies of human organs and tissues that are used for diagnosis and surgical planning. The use of tomographic images in the creation of biomodels has been arousing great interest in the medical and bioengineering area. In addition to creating biomodels by computed tomography it is also possible, using this process, to create mathematical models to perform computer simulations and analyses of regions of interest. This paper discusses the creation of a biomodel of the skull-mandibular region of a patient from CT for study and evaluation of efforts in the area of the temporomandibular joint (TMJ) aiming at the design and development of a TMJ custom prosthesis. The evaluation of efforts in the TMJ region due to the forces of mastication was made using the finite element method and the results were corroborated by comparison with mandibular models studied in similar works.

\section{Introduction}

The importance of using biomodels in surgical procedures is the possibility of visualizing a replica of the patient's anatomy, which allows the simulation of the surgical technique from that replica, and it also allows improved doctor-patient communication as well as the possibility of development and manufacture of custom prosthetic implants. However obtaining three-dimensional (3D) computer models of the bone regions is not an easy task, mainly due to the complexity of the geometry of these regions [1-3].

One of the problems in obtaining reliable models of human anatomical regions is the variation of the tissues, structures, or organs of each human individual. One way to solve this problem would be the development of appropriate tools and methods for generating three-dimensional images coming from the patient himself, for example, of the computed tomography [2]. For this, a specific software should generally be used (InVesalius) that enables the analysis of twodimensional images generated by computed tomography and later using these images to reconstruct the three-dimensional spatial model $[3,4]$.

The main software in the market today, similar to InVesalius software, which was used in this study to generate threedimensional models, are Analyze software from Mayo Foundation, ScanIP from Simpleware, Mimics from Materialise, and SolidView from C2C Technologies [5]. 
Originally the two-dimensional image of CT is represented by cutting sections of an object (patient). From the scanned flat images, specific algorithms are used to generate a $3 \mathrm{D}$ surface. The $3 \mathrm{D}$ surface can be salved in small triangles geometries known as STL format [4-6].

The volumetric reconstruction in InVesalius through the radiological two-dimensional images is done by the technique of stacking and interpolation. Using information from the DICOM files and applying the stacking and interpolation technique the result is a three-dimensional array of elements known as voxel (3D representation of a pixel). With information contained in the voxel matrix and the use of the Raycasting technique, the three-dimensional model is generated and visualized in InVesalius.

The techniques for segmentation used in the generation of the three-dimensional image in InVesalius software are applied through algorithms by the boundary, threshold, and region growth methods. The work of Santa Barbara [3] describes in detail all the techniques and characteristics of InVesalius software.

The computed tomography (CT) and other medical diagnostic tools, such as magnetic resonance imaging (MRI) and ultrasonography (US), to generate three-dimensional images have already been used to create models of biomechanical organisms that are more representative, as they enable more realistic development of $3 \mathrm{D}$ geometric image of the region of interest $[3,5]$. The generation of computer models is the first step for the study, evaluation, and analysis of structural biomodel behavior involving, inclusive, the dynamic forces of the region of interest [2].

The model can be used to simulate and develop customized implants combined with new technologies machinery. Three-dimensional printing technology coupled with three-dimensional graphic modeling and CNC machining technologies are enabling the creation of high-quality products being considered a bustling and emerging process. The use of this new technology machinery, as well as speedup production, allows significant savings in the production costs. For all of these reasons, manufacturers increasingly are choosing these production methods [9].

A 3D model can be used in medical procedures, which has multiple advantages. This model facilitates surgical planning by visualizing the anatomic characteristics of the affected tissues, which helps the surgeon gain better insight into the anatomic situation and obtain a more predictable result. It is important to use the $3 \mathrm{D}$ model to check the concordance between the prostheses and the defect before surgery [10].

The development of computer-aided design and computer-aided manufacturing (CAD-CAM) procedures has introduced automated fabrication processes. Thereafter, additive manufacturing was also introduced for the fabrication of prostheses [11].

In the medical area biomodels are being increasingly used for surgical planning and diagnosis, and more recently they are used for the development of customized implants. In a patient's customized way biomodels are created through the computerized tomographies or magnetic resonances. The medical images are manipulated in specific software allowing the generation of a three-dimensional image of the structure of interest (bone, tissue, or organs). The threedimensional image created is used to fabricate the physical model (biomodel) usually by the additive manufacturing process (rapid prototyping) $[12,13]$.

The functions of the maxilla are important to enable a perfect functioning of human chewing and also to the development of speech. A new technique for the reconstruction of the patient's maxilla is currently being used in jaw surgery. In this new technique biomodels are used to simulate surgery previously through use of the computers (virtually) as well as through physical models built on three-dimensional printers. For example, a defective jaw can be corrected by mirroring the unaffected side of the patient over the problem side and thereby creating a custom implant. This computational technique allows more precise and optimized results for surgery [14].

Computer-assisted design and manufacturing techniques combined with intraoperative navigation have been widely used for various craniomaxillofacial surgeries. Preoperative designing and intraoperative navigation can provide additional accuracy and safety during surgical reconstruction, with improved clinical outcomes [15].

This paper discusses the generation and reconstitution of a three-dimensional image of the mandibular region of a patient presenting the wear of condylar head caused by TMD (Temporomandibular Joint Diseases). In this work we present the creation of a mathematical model of the TMJ region and the analysis of the efforts in the model using the finite element method. In addition, we also discussed the alteration of the articular disc material to show the impact of this alteration in the calculation of stress and strain. The reconstruction of the condylar head of the jaw that had geometric imperfections due to wear was made, redesigning the missing pieces and using appropriate image filters that make it possible to reconstruct the empty spaces caused by the pathology.

\section{Materials and Methods}

The TMJ biomodel discussed in this article was created from the images of CT of the skull of an individual obtained in a Cone Beam CT scanner i-CAT. The files of the images in DICOM format were provided by Biomanufacturing Laboratory (INCT-BIOFABRIS).

All parameters used in the tomograph followed the standard procedure for generation of DICOM files. From the computed tomography 576 two-dimensional images in grayscale in the size of $800 \times 800$ pixels were generated. The thickness of slices generated from the computed tomography was $0.30 \mathrm{~mm}$.

DICOM is a set of standards for the treatment, storage, and transmission of medical information (medical images) in an electronic format, structuring a protocol.

The DICOM format aims to standardize the medical images so that they can be exchanged between laboratories, hospitals, and doctors' offices that will make use of these images. This format was created in the 1980s by the National Electrical Manufacturers Association (NEMA) and by the 
American College of Radiologists (ACR) and enables the connectivity of health systems to radiological imaging [5].

The DICOM (Digital Imaging and Communications in Medicine) files consist of a header with various information about the examination, patient, equipment used, among other information, and the image acquired by the medical equipment, which can be MRI, CT, or ultrasonography [3].

The DICOM protocol established a standard format for data output, which should be followed by all manufacturers of medical imaging devices. Its creation, therefore, facilitated the acquisition of images necessary for the creation of virtual models [3].

For conversion of TC files into a 3D image the InVesalius software was used to enable the generation of threedimensional surface in STL format. The correction of the imperfections of the generated image and smoothing and separation of the region of interest were made using the Rhinoceros and MeshLab software, and the image was exported in an IGES file extension. To repair the STL triangle mesh and to assemble the components of the model, was used the SolidWorks software. The InVesalius, Rhinoceros, SolidWorks, and MeshLab software were installed on a PC with Intel Pentium P6200 processor, $2.13 \mathrm{GHz}$ and $4 \mathrm{~GB}$ of RAM, which uses the Windows operating system Seven Home Basic.

Later the IGES file extension was imported of ANSYS software in the Workbench interface and a finite element model of the individual's jaw using the solid tetrahedral element was created.

The boundary conditions assumed, material properties, and muscle forces applied in the model were obtained from the literature $[8,16-21]$. In the model all the materials were considered linear.

The inferred load of $1000 \mathrm{~N}$, distributed among the main muscles of human chewing, used in this work to perform the simulation of stress and strain, is a critical condition of chewing and was applied to verify the response of the mathematical model to the extreme conditions of application of forces, coherent with human chewing [22-24].

It is important to note that the articular disc modeled in this work is not a faithful representation of the actual anatomy of the human articular disc, but it is a better condition than the simple omission of this region in models that study the efforts in the TMJ region.

The elastic modulus of adult compact bone was considered $15 \mathrm{GPa}$ and the Poisson coefficient equal to $0.30[16,18$, 21]. The model that has the articular disc of elastic material shows values for the modulus of elasticity equal to $150 \mathrm{MPa}$ and Poisson coefficient equal to 0.49 [25].

2.1. Generation of Jaw 3D Model from CT. The CT data in its original form (raw data) are not suitable for model generation; there is a need for preprocessing and data processing.

The use of the model generated by the radiological images in the raw CT state is not indicated due to inconsistencies in the creation of the 3D model in InVesalius software. When using the software to perform a structural analysis by the finite element method, the separation of the region of interest must be performed in complementary software, facilitating the manipulation of the model and eliminating "noise" in the generated image. Unwanted geometries can be excluded to improve the virtual model and the surface smoothing process must also be performed to make the model, already exported in the STL format in InVesalius software, less discretized and more continuous.

The files of the individual skull CT in DICOM format were imported to InVesalius software and this preprocessing step is crucial to define the parameters for model generation. The generation of the 3D image involves the definition of how many and which slices (flat images) of CT will be used. A greater number of slices (images) improve the resolution of the model but slow down the process. The choice of which and how many slices will be used depends on balance between resolution and processing time. The option in this work was the resolution and all CT images were used for 3D image creation. The choice of the type of material used in the model is directly related to the region to be scanned, since human tissues are composed of cells grouped together to perform a particular function, for example, bone tissue. To model the region of TMJ the tissue chosen was the Compact Bone Adult defined in InVesalius software. This choice was used because of patient characteristics and to present good results during the generation of the image. The preprocessing tool InVesalius software allows manually clearing the twodimensional images and selecting the region of interest to study; it also allows eliminating unwanted areas, highlighting areas that might be faulty, or even recreating defective areas, redesigning the image.

In the present case the mandibular condyle has wear due to pathologies of the TMJ and was rebuilt using, in addition to InVesalius, the image manipulation software known as MeshLab (Figure 1).

The reconstruction of the condylar head area was made redrawing the image on each slice flat (2D) stemmed of CT in the InVesalius software. After meshing of three-dimensional triangles in STL format in InVesalius image filters were used which led to the improvement of the condylar head repair jaw. The three-dimensional mesh STL was exported to MeshLab software and surface smoothing filters were used for the position adjustment of the STL mesh points, improving the image preview $[2,3]$. Then it used a filter for decimation of the triangular mesh, which reduced the number of triangles used in the mesh $[2,3]$.

Some distortions in the model can be created due to the use of smoothing and decimation filters. In the case of the process used in this study, the use of the filters was necessary to reconstruct the patient's condyle and to obtain a more homogeneous geometry and also due to software and hardware limitations. These types of filters should be used with care so as not to distort the geometric model excessively and should never be used as a standard procedure.

Through the 3D image of the mandible in STL format the file was exported to the software Rhinoceros. In Rhinoceros plug-in was used known as MeshToSolid for converting a mesh of triangles into a volume NURBS format and, finally, the $3 \mathrm{D}$ model was defined in the IGES format that can be read in most analysis software by finite element method. 


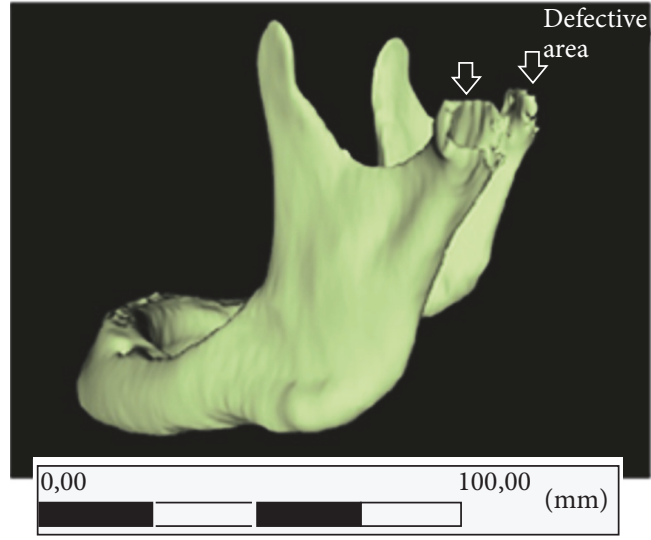

(a)

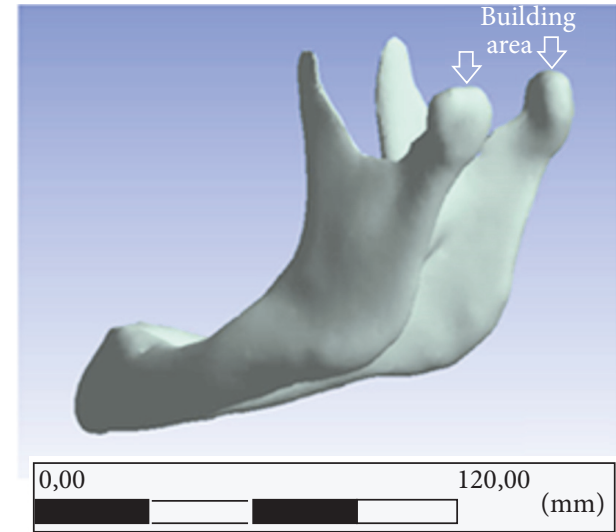

(b)

FIGURE 1: The patient's jaw with condylar defect (a) and subsequently reconstructed (b).

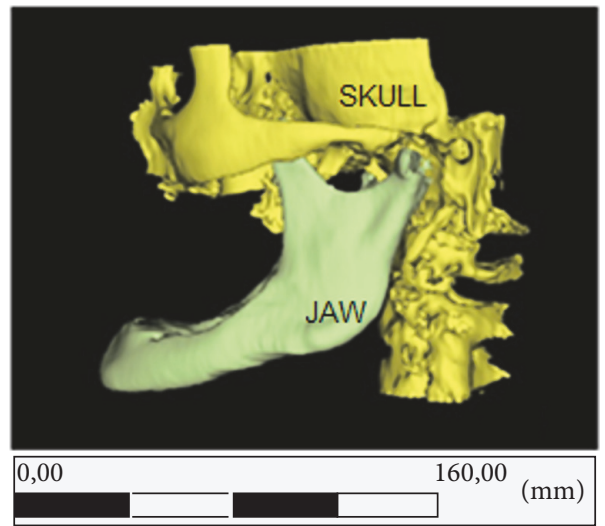

(a)

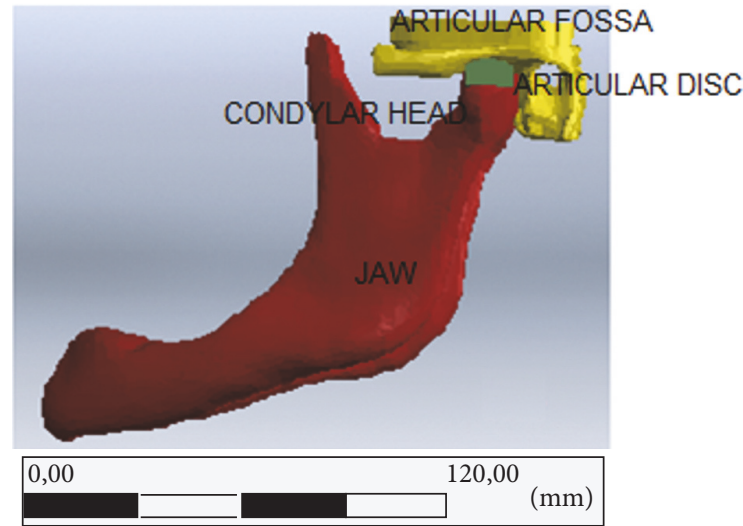

(b)

FIgURE 2: Image of skull-mandibular region of the patient (a) and (b) model proposed by the separation of the TMJ region in three components, jaw (red), articular fossa (yellow), and articular disc (green).

An important aspect to be highlighted at this stage is to observe the shape of the elements in the $3 \mathrm{D}$ mesh; that is, there may be degenerate elements in the $3 \mathrm{D}$ model. This problem was solved by using a tool called diagnostic import implemented in SolidWorks that scans the mesh of triangles locating disconnected areas and offers automated solutions for the union of the three-dimensional solid surface.

Not always can degenerate elements be corrected with the diagnostic import from SolidWorks. To solve this problem there is a free SolidWorks plug-in "Scan to 3D" that can be used as an option for correction, because it has some tools to clean and correct a mesh and make a better NURBS model. ANSYS “virtual topology” tool can solve problem geometries and enable finite element mesh of better quality. ANSYS DesignModeler and ANSYS SpaceClaim, when available, are great tools to correct irregular geometries and provide a more realistic modeling.

Figure 2(a) shows the complete three-dimensional image of CT, generated in InVesalius, involving all bone elements of the skull-jaw region. To evaluate and analyze the set of TMJ we performed the separation and assembly of the components of the model from the image of the skull-jaw generated region. Figure 2(b) shows the proposed model in which the TMJ area was separated in three components, called jaw (highlighted in red), articular fossa (in yellow), and articular disc (highlighted in green).

The separation was done with reference to Figure 3(a) which shows an illustration in real anatomy of cutting the TMJ area of the patient, wherein the three main components of the temporomandibular joint region can be seen beyond cavities in the upper and lower disc joint where synovial fluid is present. The articular fossa was created from the patient's skull, which was carried out by cuts in the cranial structure allowing separation in two distinct parts in the virtual model which later were positioned in the area of the condylar head, above the articular disc. The articular disc is the interface between the skull and the condyle head; to create this component we carried out, in Rhinoceros software, Boolean operations between the two structures (jaw and skull) and the resulting intersection generated the articular 

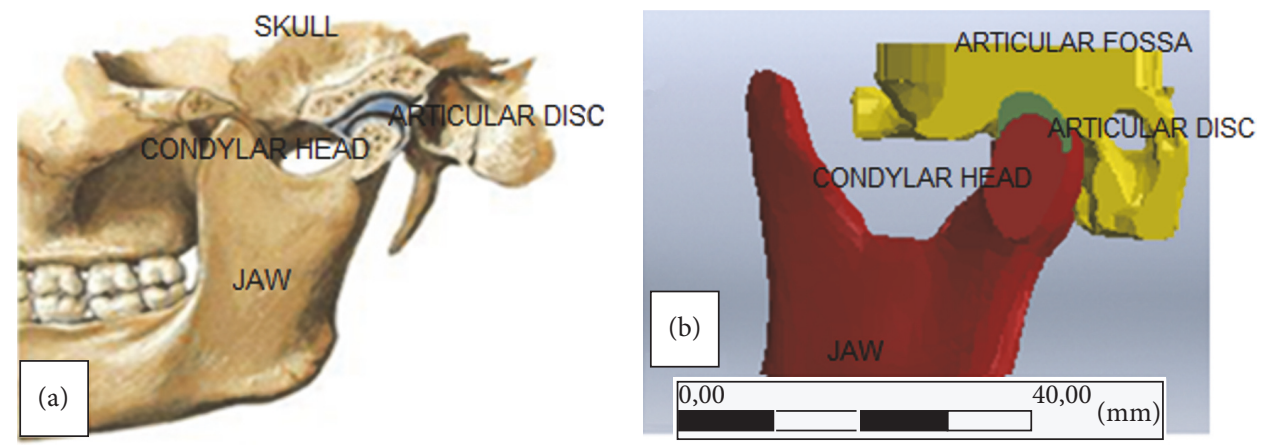

FIgURE 3: Human anatomy illustrated by Netter (a) and cut model created showing the joint disc (b). Source: adapted from Netter [7].

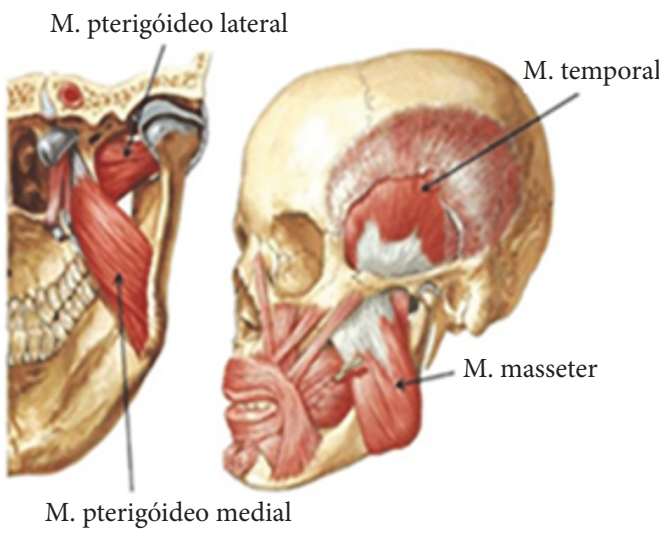

(a)

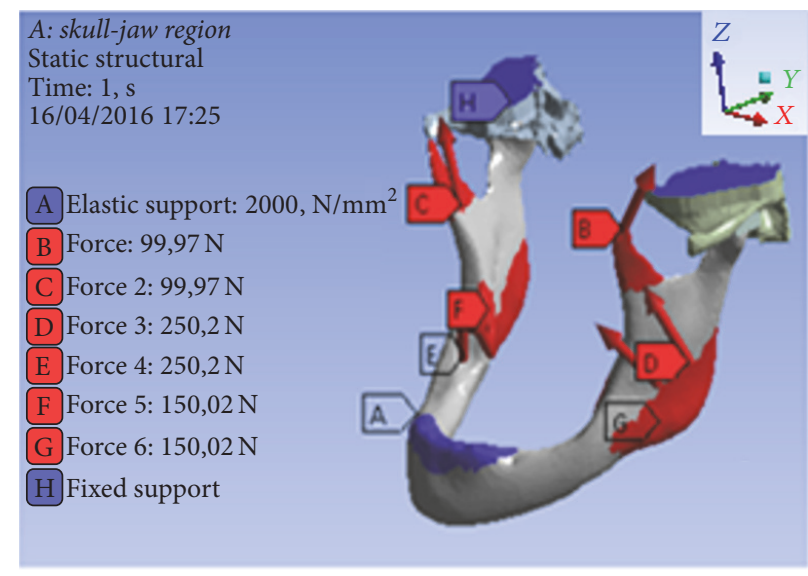

(b)

Figure 4: Main muscles of mastication in (a) and boundary conditions applied to the virtual model (b). Source: adapted from Sobotta and Reinhard [8].

disc of the computer model. This structure was considered as the union of the existing articular disc in human anatomy and synovial fluid which lubricates TMJ, discussed by Netter [7].

Figure 3(b) shows a cut of TMJ region of the virtual model created from CT; in this model the cavities of synovial fluid were disregarded, considering only the articular disc at the interface between the skull and jaw.

After defining the final model of the TMJ region, the file was exported to the ANSYS software to perform the structural analysis using the finite element method.

2.2. Finite Element Analysis of the Jaw. The analysis by the finite element method to calculate and study the stress and strain in the skull-jaw region due to the forces involved in the mastication was performed to generate knowledge about the efforts and thus to allow the development of customized prostheses through the acquired knowledge. The Finite Element Model of TMJ in this study was generated in ANSYS commercial software using tetrahedral solid elements (Solid 187).

The forces applied in the model were defined based on the performance of the main muscles involved in human mastication: the masseter, medial pterygoid, and temporal. The arrangement of muscles and the terminations is illustrated in Figure 4(a). The loads were inferred from the order of $1000 \mathrm{~N}$ distributed in respective inserts of the jaw muscles symmetrically in relation to the left and right side of the jaw. The directions of the forces applied and muscles positions were defined on the basis of the work of Bertol [20] and AlAhmari et al. [21]. The masseter muscle received a $500 \mathrm{~N}$ of load, the medial pterygoid muscle load of $300 \mathrm{~N}$, and the temporalis muscle load of $200 \mathrm{~N}$ [22-24].

Table 1 shows the directions of forces vectors (decomposition in the $x-, y$-, $z$-axis) with respect to the origin coordinate axis shown in Figure 4(b), representing the muscle reaction during the act of chewing.

The rigid constraints of the mandible and cranium were approximated by high stiffness springs (position A in Figure 4(b)) symmetrically in incisor teeth and a fully rigid fixation (position $\mathrm{H}$ in Figure 4(b)) at the top of the articular fossa. All boundary conditions applied to the model, and positions and orientations of muscle forces and constraints considered are shown in Figure 4(b).

The characteristics attributed to the high rigidity springs (spring constant) were determined through simulation and verification of the results of strain in the model itself of the 
TABLE 1: Direction in space of the force vectors representing each muscle of mastication and its magnitude. Source: adapted from Bertol [20].

\begin{tabular}{lcccc}
\hline Muscles & $\begin{array}{c}x \text {-axis } \\
(\mathrm{N})\end{array}$ & $\begin{array}{c}y \text {-axis } \\
(\mathrm{N})\end{array}$ & $\begin{array}{c}z \text {-axis } \\
(\mathrm{N})\end{array}$ & $\begin{array}{c}\text { Magnitude } \\
(\mathrm{N})\end{array}$ \\
\hline Masseter right & 104,7 & $-51,7$ & 221,2 & 250 \\
Masseter left & $-104,7$ & $-51,7$ & 221,2 & 250 \\
Temporal right & $-50,0$ & 22,1 & 83,7 & 100 \\
Temporal left & 50,0 & 22,1 & 83,7 & 100 \\
Medial pterygoid right & 55,8 & $-72,9$ & 118,6 & 150 \\
Medial pterygoid left & $-55,8$ & $-72,9$ & 118,6 & 150 \\
\hline
\end{tabular}

skull-mandibular region and compared to the results of strain found in simulations using fully rigid supports, which is a finding that forms an ideal value for high stiffness springs.

For mesh model elements generation we used automatic generation with 1.0 to $3.0 \mathrm{~mm}$ size elements, tetrahedral shape, and mathematical algorithm Patch Independent type. Later we conducted convergences evaluation from simulations of the resulting stress.

The Patch Independent algorithm is not the best choice for generating finite element meshes for structural analysis; however due to the existence of complex geometries in the model this option was really necessary.

The contacts between the structures of the virtual model were performed using standard ANSYS Workbench bonding elements.

Figure 5 shows the mesh of the finite element model of the skull-jaw system.

The element used was solid tetrahedral type (Solid 187) and the average processing time was 913.54 seconds. The interactions for solution of the problem were stopped when an error in the variation of the computational calculation stress was lower than $1.0 e-08$. The numbers of nodes and elements of the model were as follows: 626,590 and 417,320.

\section{Results and Discussion}

The analyses of the efforts in the model were performed considering two conditions, a first analysis in which all skulljaw assembly components have the same stiffness and another condition that the components have different stiffness. In the first proposed analysis, it was considered that the material of the skull-jaw assembly components has similar mechanical properties to adult compact bone, elastic modulus equal to $15 \mathrm{GPa}$, Poisson's ratio equal to $0.30[16,18,21]$.

Figure 6 shows the result of the stress and strain distribution of the model. Analyzing the simulation results in terms of von-Mises stress, the maximum stress observed is in the order of $33.20 \mathrm{MPa}$ and occurs in the lower part of the neck of the condylar head (red region in Figure 6(a)). The observed maximum deformation is on the order of $0.29 \mathrm{~mm}$ on top of the temporal muscle attachment area in the mandible (red region in Figure 6(b)).

In the second analysis, the articular disc was found with mechanical properties similar to elastic materials. This

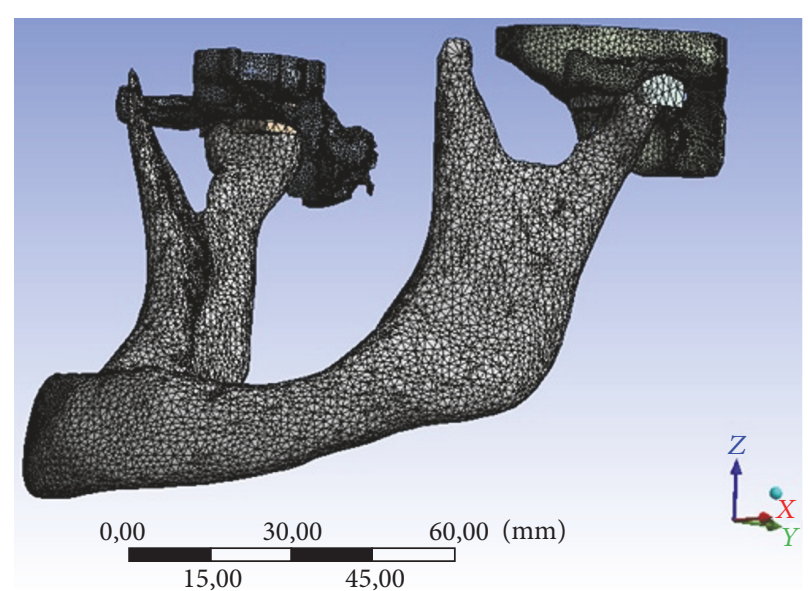

FIGURE 5: Tetrahedral mesh generated by ANSYS Workbench software.

consideration was made due to the gelatinous characteristic of articular disc that is evidenced in books and papers already published $[7,8]$. The area between the condylar head and the articular fossa was considered as a single interface with elastic characteristics and the used material properties were elasticity modulus of $150 \mathrm{MPa}$ and Poisson's ratio equal to 0,49 [25].

In the second analysis the set is modeled with the articular disc as elastic structure and Figure 7 shows the results of stress and strain distribution of the model. The maximum stress observed was $27.94 \mathrm{MPa}$ (Figure 7(a)) and the maximum deformation was $0.28 \mathrm{~mm}$ (Figure 7(b)).

In this model, you can see that larger deformations occur in the articular disc relative to the bone disc model, as would be expected, and stresses had lower magnitudes compared to the first analysis.

The comparison of the values obtained shows that they are consistent with those observed in other works on the stress-strain analysis of the human jaw [18-20]. In the work of Tie [18] the maximum stress was $25 \mathrm{MPa}$ for healthy jaw model (completely full), while for Mahdian et al. [19] the maximum stress is around $60 \mathrm{MPa}$, but in his study the model comprises an alloplastic implant titanium, applied to the medial part of the jaw, which probably led to higher stress. For Bertol [20] the maximum von-Mises stress, considering a healthy jaw, is around $20 \mathrm{MPa}$. In the studies quoted in this paragraph, muscle forces (loads) applied in the respective models are slightly below those applied in the model of this article, which explains the small positive variation of the results of von-Mises stress presented here. It is also important to emphasize that each individual has geometric characteristics (anatomical) specific for their jaws and this makes the differences of the simulation results found in studies.

The calculated maximum stress shown in Figures 6 and 7 , located at the bottom of the neck of the condylar head, does not reach the limit of human bone of the order of 130-190 MPa [16]. 


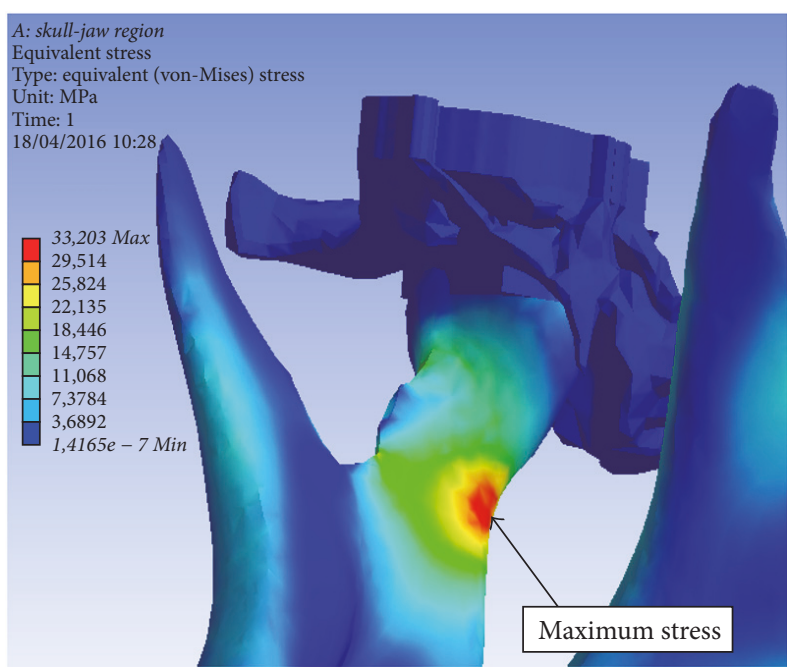

(a)

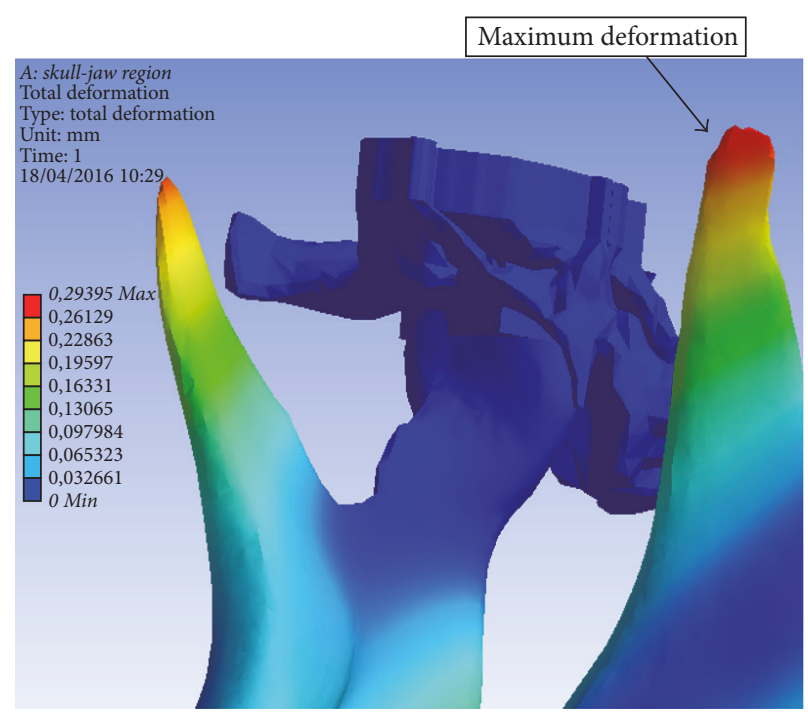

(b)

FIGURE 6: Stress distribution (a) and strain distribution (b) in skull-jaw assembly with rigid articular disc.

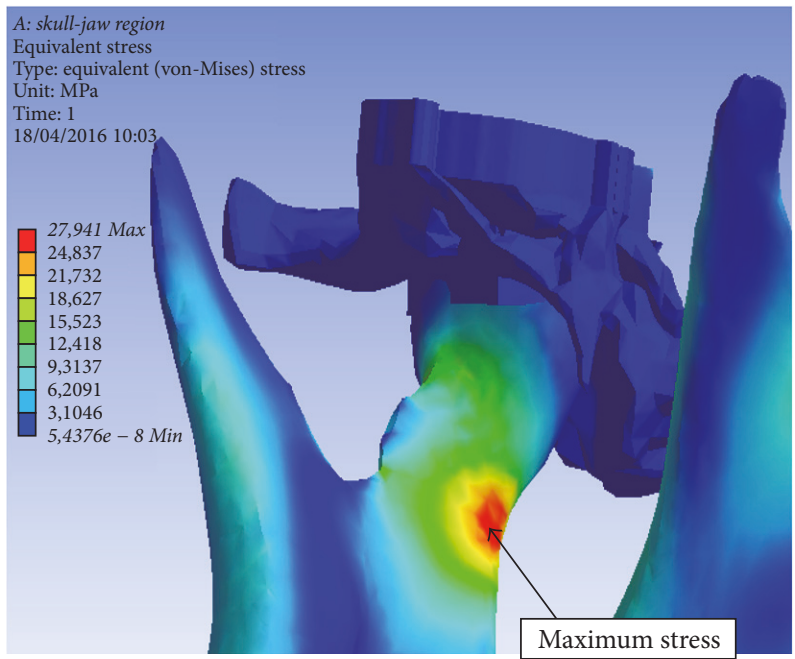

(a)

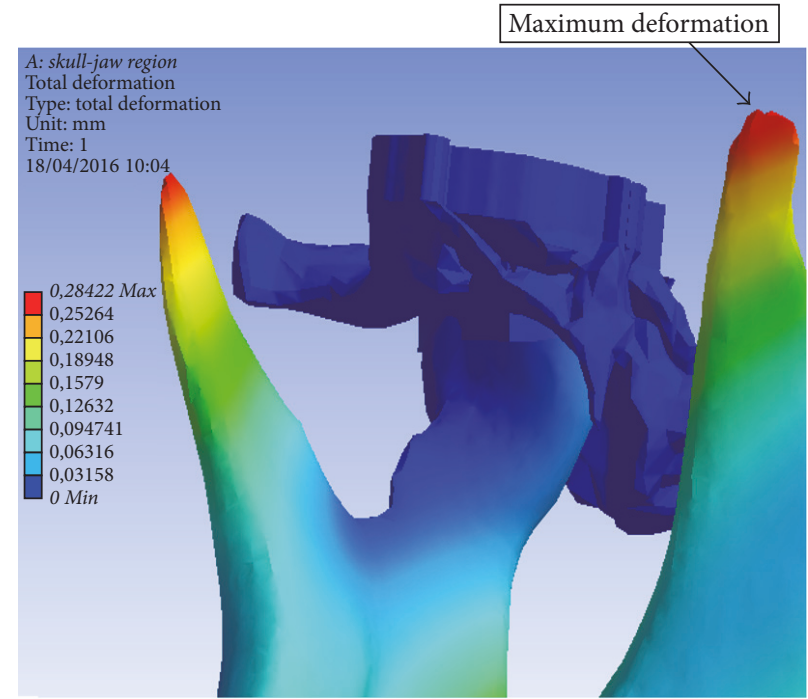

(b)

FIGURE 7: Stress distribution (a) and strain distribution (b) in skull-jaw assembly with elastic articular disc.

For the model with articular disc with elastic properties as for the model with articular disc with bony properties the calculated tensions are below the yield stress of the human bone which suggests a safe structural condition. The results obtained by changing the type of articular disc material did not bring discrepant changes between tests for the loading conditions proposed. However, it is believed that, for dynamic analyses involving fatigue effects or other dynamic loads, the disc condition with elastic properties is more representative. It is also important to emphasize that human bone has bioactive properties that were disregarded in the simulation performed in this paper. The bioactive properties of human bone give it greater resistance due to micro natural mechanisms. In the simulation they were considered inert materials, that is, materials that show no bioactivity.

\section{Conclusion}

This paper presents a three-dimensional modeling of the TMJ of an individual from the CT images of the skull-jaw of a patient's system with wear of the temporomandibular joint and the structural analysis of the efforts in the jaw was later made by the finite element method.

The main issues addressed for the generation of threedimensional model were converting the files originally in DICOM format for the STL format and the use of specific 
mathematical tools to eliminate elements irregularly shaped and surfaces and discontinuous edges and defects in image formation and so generate a 3D solid in IGES extension, compatible with all major finite element software. Another important aspect is the process of computational reconstruction of the condylar head that could be used to obtain a custom implant for repair of eroded or degenerated bone structure.

The simulation of two conditions for representing the interface between the condylar head and the articular fossa (articular disc) showed the versatility that the designer has available for developing customized implants and may depict the synovial fluid and real articular disc with elastic characteristics, which can lead to various reanalysis involving different efforts. It is also important to note that, comparing the two models created in this work (elastic articular disc and bone articular disc), small differences were found in the results of stresses and strains, indicating that simple models used in studies present in the literature also provide consistent results and can be used in the simulations proposed.

One limitation of the model is not to consider the different densities and types of structures of the jaw bone and of the skull bone. The model created in InVesalius software and exported in the STL extension assumes a totally homogeneous structure.

The importance of this work in the use of buccomaxillary surgeries comes through the creation of a model of the TMJ region that provides stress and strain values of this region, allowing an analysis of possible prostheses to be used in patients with pathologies in the studied area. In addition, it is possible to construct the model in $3 \mathrm{D}$ printer to perform physical simulations of surgeries embedding implants in the printed model (biomodel) and simulating the surgical act previously (surgical study).

This work created a computer model of the skull-jaw region obtained based on the patient's own features from CT. This opens a large window of opportunity for simulating and developing custom prostheses using rapid prototyping metals equipment as described by Gunsoy and Ulusoy [9], Shan et al. [10], Kim et al. [11], and Tarsitano et al. [14].

And, finally, it is important to note that InVesalius software is free and contains the essential tools for the process of three-dimensional imaging from CT.

\section{Conflicts of Interest}

The authors declare that there are no conflicts of interest regarding the publication of this paper.

\section{Acknowledgments}

The authors would like to thank The National Institute of Science and Technology in Bioengineering (INCT-BIOFABRIS) and PROCAD project for the financial support.

\section{References}

[1] M. I. Meurer, E. Meurer, J. V. L. Silva et al., "Aquisição e manipula ção de imagens por tomografia computadorizada da região maxilofacial visando à obtenção de protótipos bio-médicos," Radiologia Brasileira, vol. 41, no. 1, pp. 49-54, 2008.

[2] Jóia Filho P and E. A. Capello Sousa, "Reconstrução e geração de malhas em estruturas biomecânicas tridimensionais para análise por elemento finitos," Revista Brasileira de Engenharia Biomédica, vol. 25, no. 1, pp. 15-20, 2009.

[3] A. Santa Barbara, Processamento de imagens médicas tomográficas para modelagem virtual e física: o software In Versalius [thesis], Universidade Estadual de Campinas, São Paulo, Brazil, 2006.

[4] N. Celebi, E. C. Rohner, J. Gateno et al., "Development of a mandibular motion simulator for total joint replacement," Journal of Oral and Maxillofacial Surgery, vol. 69, no. 1, pp. 66-79, 2011.

[5] C. B. L. Ulbrich, Inspeção por digitalização em aplicações de prototipagem rápida na medicina [thesis], Campinas: Universidade Estadual de Campinas, 2007.

[6] J. V. Silva, A. Santa Barbara, M. F. Gouveia, M. C. Reis, and C. A. Zavaglia, "Aplicación del prototipaje rápido al tratamiento de defectos cráneo faciales," Revista CENIC: Ciencias Biológicas, vol. 37, no. 3, pp. 214-218, 2006, Recuperado de http://www .redalyc.org/articulo.oa?id=181220529019.

[7] F. H. Netter, Atlas de Anatomia Humana, Elsevier, Rio de Janeiro, Brazil, 6th edition, 2015.

[8] J. Sobotta and P. Reinhard, Atlas da Anatomia Humana: Cabeça, Tronco, Membros Superior, Guanabara Koogan, Brasil, 22nd edition, 2006.

[9] S. Gunsoy and M. Ulusoy, "Evaluation of marginal/internal fit of chrome-cobalt crowns: direct laser metal sintering versus computer-aided design and computer-aided manufacturing," Nigerian Journal of Clinical Practice, vol. 19, no. 5, pp. 636-644, 2016.

[10] X. F. Shan, H. M. Chen, J. Liang, J. W. Huang, and Z. G. Cai, "Surgical reconstruction of maxillary and mandibular defects using a printed titanium mesh," Journal of Oral and Maxillofacial Surgery, vol. 73, no. 7, pp. 1-9, 2015.

[11] E. H. Kim, D. H. Lee, S. M. Kwon, and T. Y. Kwon, "A microcomputed tomography evaluation of the marginal fit of cobaltchromium alloy copings fabricated by new manufacturing techniques and alloy systems," The Journal of Prosthetic Dentistry, vol. 22, no. 6, pp. 30352-30353, 2017.

[12] M. A. Larosa, A. L. Jardini, C. A. C. Zavaglia, P. Kharmandayan, D. R. Calderoni, and R. M. Filho, "Microstructural and mechanical characterization of a custom-built implant manufactured in titanium alloy by direct metal laser sintering," Advances in Mechanical Engineering, vol. 6, pp. 8-16, 2014.

[13] A. L. Jardini, M. A. Larosa, C. A. de Carvalho Zavaglia et al., "Customised titanium implant fabricated in additive manufacturing for craniomaxillofacial surgery: this paper discusses the design and fabrication of a metallic implant for the reconstruction of a large cranial defect," Virtual and Physical Prototyping, vol. 9, no. 2, pp. 115-125, 2014.

[14] A. Tarsitano, S. Battaglia, L. Ciocca, R. Scotti, R. Cipriani, and C. Marchetti, "Surgical reconstruction of maxillary defects using a computer-assisted design/computer-assisted manufacturingproduced titanium mesh supporting a free flap," Journal of Cranio-Maxillofacial Surgery, vol. 44, no. 9, pp. 1320-1326, 2016.

[15] W.-B. Zhang, C. Mao, X.-J. Liu, C.-B. Guo, G.-Y. Yu, and X. Peng, "Outcomes of orbital floor reconstruction after extensive maxillectomy using the computer-assisted fabricated individual titanium mesh technique," Journal of Oral and Maxillofacial Surgery, vol. 73, no. 10, pp. 1-15, 2015. 
[16] C. H. Turner and D. B. Burr, "Basic biomechanical measurements of bone: a tutorial," Elsevier: Bone, vol. 14, no. 4, pp. 595608, 1993.

[17] J. Parthasarathy, “3D modeling, custom implants and its future perspectives in craniofacial surgery," Annals of Maxillofacial Surgery, vol. 4, no. 1, pp. 18-19, 2014.

[18] Y. Tie, “Three-dimensional finite-element analysis investigating the biomechanical effects of human mandibular reconstruction with autogenous bone grafts," Journal of Cranio-Maxillofacial Surgery, vol. 34, no. 5, pp. 290-298, 2006.

[19] N. Mahdian, T. Dostálová, J. Daněk et al., "3D reconstruction of TMJ after resection of the cyst and the stress-strain analyses," Computer Methods and Programs in Biomedicine, vol. 110, no. 3, pp. 279-289, 2013.

[20] L. S. Bertol, Contribuição ao estudo da prototipagem rápida, digitalização tridimensional e seleção de materiais no design de implantes personalizadosdo sul, Universidade Federal do Rio Grande do Sul, Porto Alegre, Brazil, 2008.

[21] A. Al-Ahmari, E. A. Nasr, K. Moiduddin, S. Anwar, M. A. Kindi, and A. Kamrani, "A comparative study on the customized design of mandibular reconstruction plates using finite element method," Advances in Mechanical Engineering, vol. 7, no. 7, pp. 1-11, 2015.

[22] M. M. D. Seixas, Estudo de propriedades físicas de materiais adesivos ortodônticos, Universidade Estadual Paulista, São Paulo, Brazil, 2005.

[23] K. J. Anusavice, Phillips Materiais Dentários, Guanabara Koogan, Rio de Janeiro, Brazil, 10th edition, 1998.

[24] I. R. Reynolds, “A review of direct orthodontic bonding," British Journal of Orthodontics, vol. 3, no. 2, pp. 171-178, 1975.

[25] D. R. H. Jones and M. F. Ashby, Engineering Materials 2: An Introduction to Microstructures, Processing And Design, Butterworth-Heinemann, 2005. 

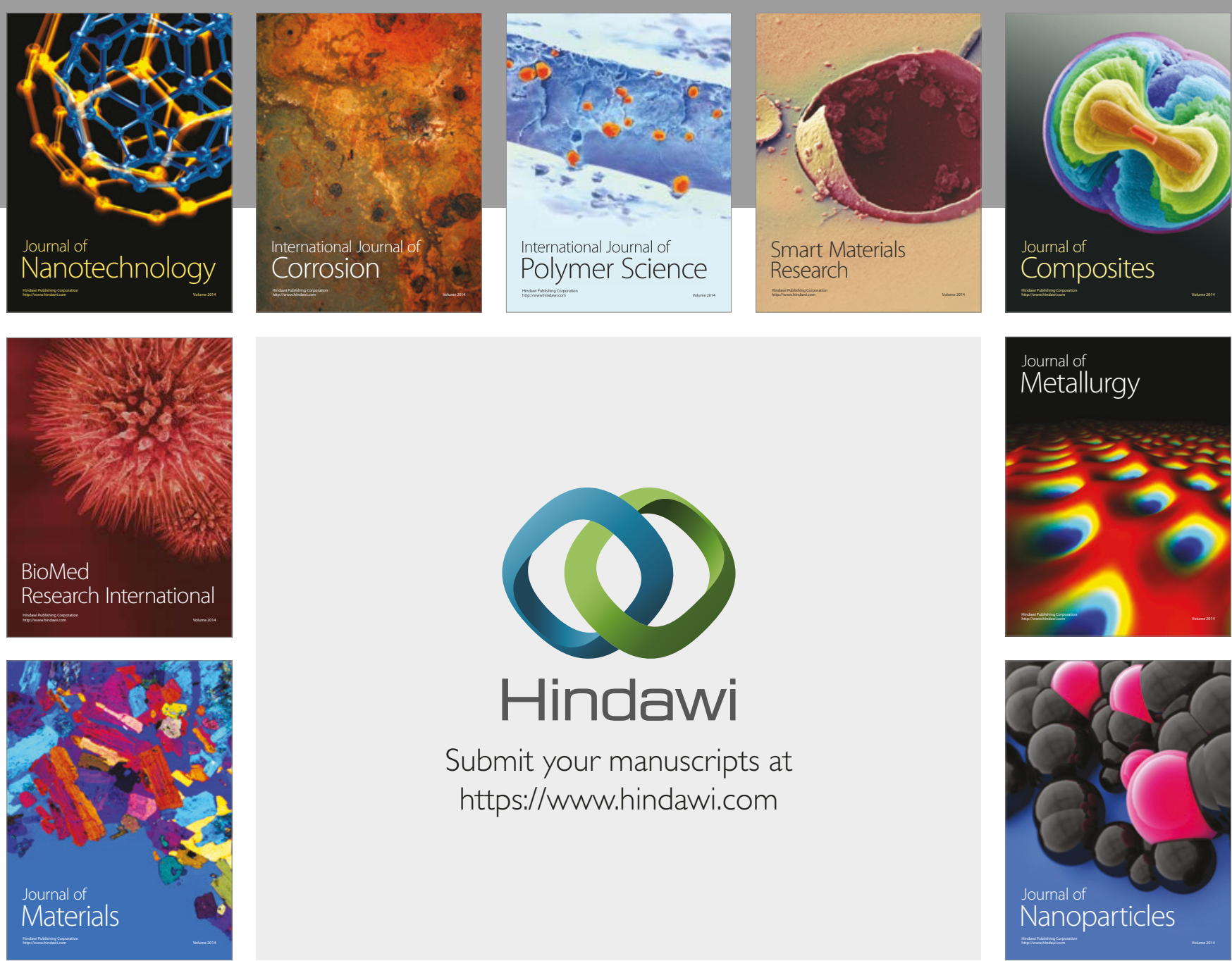

\section{Hindawi}

Submit your manuscripts at

https://www.hindawi.com
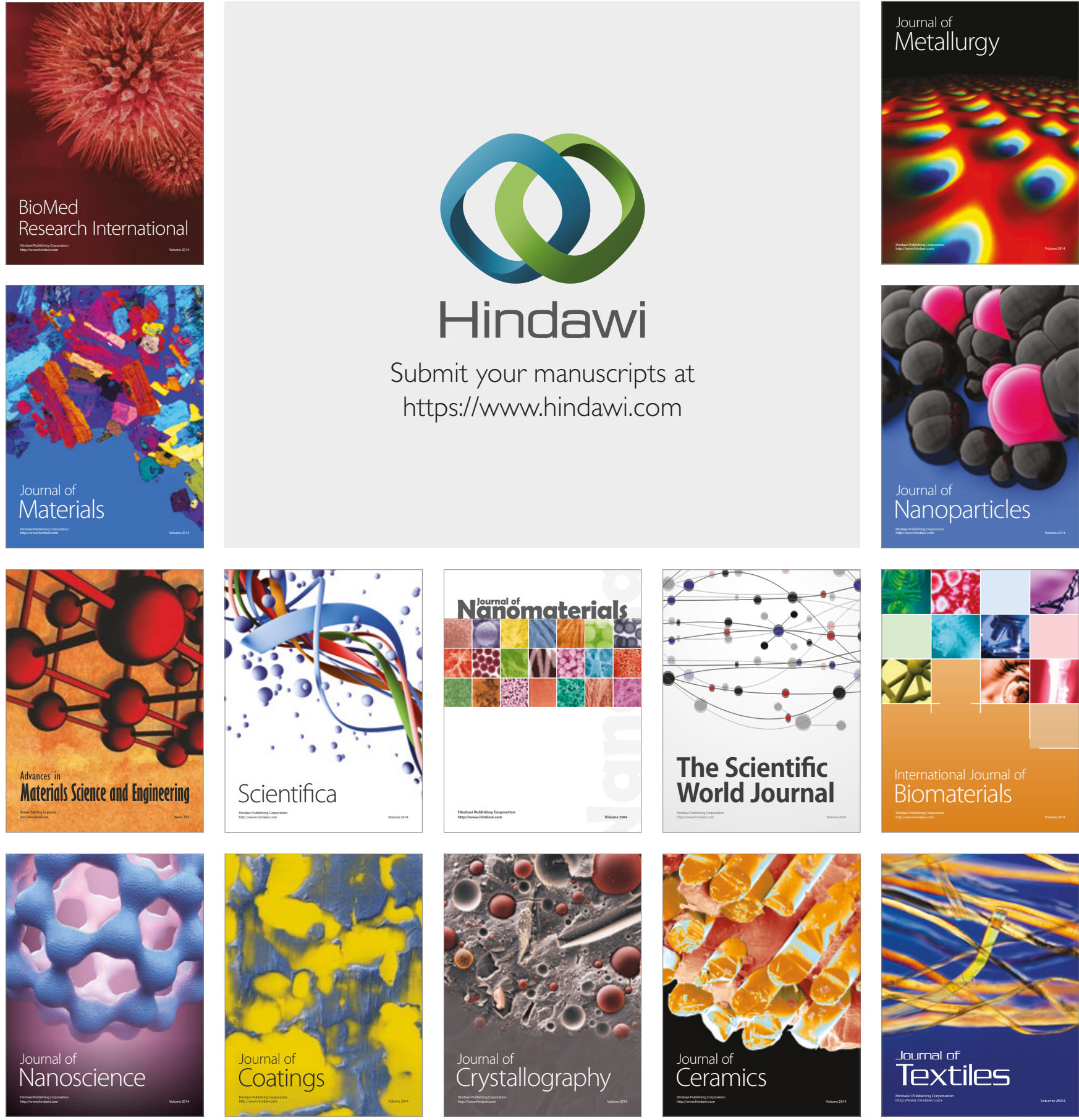

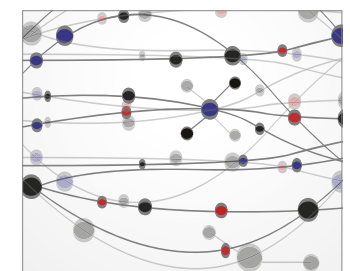

The Scientific World Journal
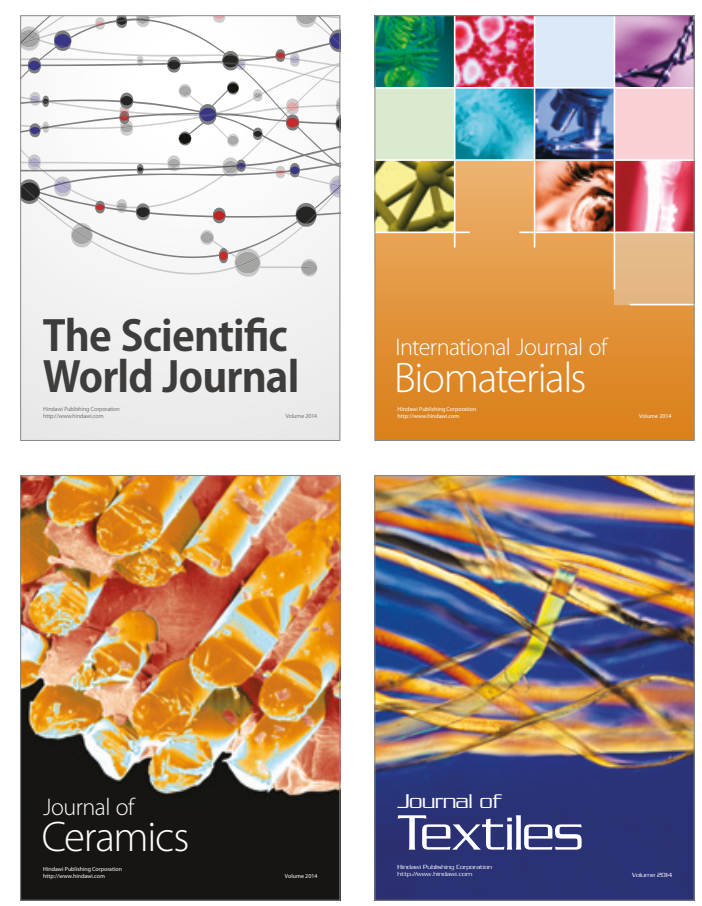